\title{
Complex and thermodynamic properties of polar liquids using time domain reflectometry
}

\author{
Shagufta Tabassum*, ${ }^{\ddagger}$ and V. P. Pawar ${ }^{\dagger}$ \\ *Department of Physics \& Electronics, Maharashtra Udayagiri Mahavidyalaya \\ Udgir, Latur 413517, Maharashtra, India \\ †Sunderrao Solanke Mahavidyalaya, Majalgaon \\ Beed 431131, Maharashtra, India \\ †tabussum.sagufta@gmail.com
}

\begin{abstract}
Received 25 July 2018; Revised 10 September 2018; Accepted 16 September 2018; Published 12 October 2018
The study of complex properties in a binary mixture of 1,2-dichloroethane (DE) and $n$-methylformamide (NMF) polar liquids has been carried out in the frequency range of $10 \mathrm{MHz}$ to $30 \mathrm{GHz}$ for 11 different concentrations using time domain reflectometry technique at $283,288,293$ and $298 \mathrm{~K}$ temperatures. Complex property of binary liquids indicates the type of distribution of the dielectric relaxation time. The Bruggeman parameter gives the information about molecular interactions within binary polar liquids. Thermodynamic parameter deals with the passing of a dipole across a potential barrier which separates the minima of energy.

Keywords: Time domain reflectometry; complex properties; Bruggeman parameters; 1,2-dichloroethane; $n$-methylformamide; thermodynamic parameters.
\end{abstract}

\section{Introduction}

For the representation of the complex permittivity data, different models are used (Debye, Cole-Cole (CC) and Cole-Davidson (CD)). Complex permittivity contains real and imaginary terms. The real term is known as static permittivity $\left(\varepsilon^{\prime}\right)$ and the imaginary terms as permittivity loss $\left(\varepsilon^{\prime \prime}\right)$. Based on the shape of complex permittivity, type of model is selected.

1,2-dichloroethane (DE) is a highly flammable, colorless liquid with sharp, harsh odor. DE is a polar binary liquid which is soluble in water. It is used to produce solvents in chemical mixture. DE used as plating agents and surface agents in industries and also for cleaning and furnishing care products. ${ }^{1}$

$N$-methylformamide (NMF) is a colorless, nearly odorless organic component. It is a water soluble organic solvent. NMF mainly used as a reagent in various organic syntheses and cellular antineoplastic agent (substance that prevents the proliferation of neoplasms) with high polar solvent. ${ }^{2}$

The complex properties of binary polar liquids have been carried out by many workers by using time domain reflectometry (TDR) technique. ${ }^{3-6}$ The DE and NMF linkages $(-\mathrm{CH} 2-\mathrm{Cl})$ and $(-\mathrm{NHC}=\mathrm{O})$, respectively, are very important functional groups in chemical industries and material science. The main objective of this paper is to derive the complex and thermodynamic parameters of DE-NMF at 283, 288, 293 and $298 \mathrm{~K}$ temperatures for 11 different concentrations.

\section{Experimental Technique}

DE (AR Qualigens fine chemicals Pvt. Ltd., Mumbai, India) and NMF (Merck Specialties, Pvt. Ltd., Mumbai, India) binary polar liquids are used without further purification. DE-NMF Liquid mixture is prepared at 11 different volume percents by increasing NMF from $0 \%$ to $100 \%$ in DE system just before the measurement. By using these volume percents, the mole fraction is calculated as

$$
x=\frac{\frac{v_{1} \rho_{1}}{m_{1}}}{\left[\frac{v_{1} \rho_{1}}{m_{1}}+\frac{v_{2} \rho_{2}}{m_{2}}\right]},
$$

where $m_{i}, v_{i}$ and $\rho_{i}$ represent the molecular weight, volume percent and density of the $i$ th $(i=1,2)$ liquids, respectively.

TDR technique is used to obtained complex permittivity spectra for binary liquids..$^{3-6}$ TDR is based on the principle of transmission line theory which helps us for studying the heterogeneities in coaxial lines in which the coaxial line is used as the communication media. The shape of incident pulse $R_{1}(t)$ is same as long as the sample cell is empty. However, the pulse shape changes if any binary liquid is inserted in the sample cell. In that case, the signal is partly reflected back from the interface $R_{x}(t)$ and partly refracted or passes through it. For the static dielectric measurements, the sample cell is placed at the end of the coaxial line as shown in Fig. 1. A step generator generates a fast rising repetitive

This is an Open Access article published by World Scientific Publishing Company. It is distributed under the terms of the Creative Commons Attribution 4.0 (CC-BY) License. Further distribution of this work is permitted, provided the original work is properly cited. 


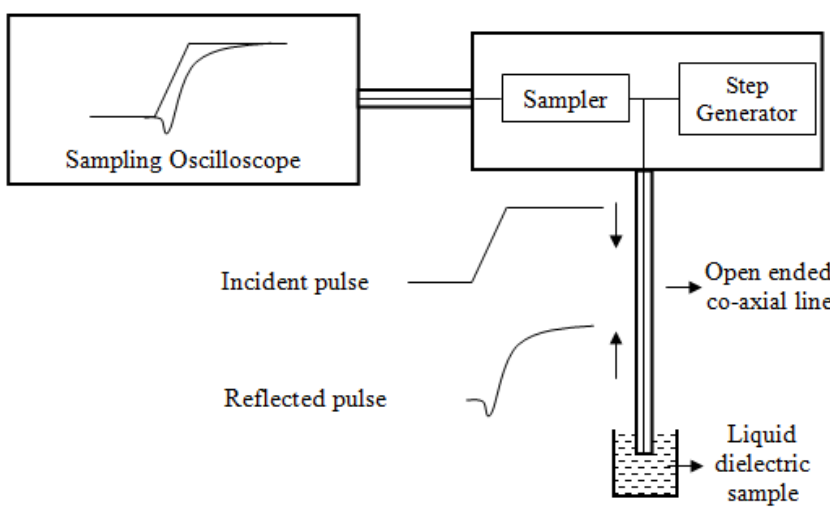

Fig. 1. Tektronix DSA8200 digital serial analyzer sampling oscilloscope.

voltage which is allowed to pass through the coaxial line and made to incident on the sample placed in the sample cell. These incident and reflected pulses are detected and displayed on the sampling oscilloscope. The sample response is the total voltage response across the dielectric material which is equal to the algebraic sum of the applied and reflected voltages $\left[R_{1}(t)+R x(t)\right]$. In this method, the entire frequency spectrum is covered at once.

The reflected pulse with and without are observed. In microwave frequency range, the time-dependent data were converted into reflection coefficient spectra $\rho *(\omega)$ by using Fourier transformation as ${ }^{7,8}$

$$
\rho^{*}(\omega)=\frac{c}{j \omega \tau}\left[\frac{p(\omega)}{q(\omega)}\right]
$$

where $p(\omega)$ and $q(\omega)$ are Fourier transforms of $\left[R_{1}(t)-R_{x}(t)\right]$ and $\left[R_{1}(t)+R_{x}(t)\right]$, respectively, $c$ is the velocity of light, $\omega$ is angular frequency, $d$ is the effective pin length and $j=\sqrt{-1}$.

The complex permittivity spectra $\varepsilon^{*}(\omega)$ were obtained from reflection coefficient spectra $\rho *(\omega)$ by using bilinear calibration $^{9}$ method. The general form of the relaxation model is given by the Havriliak-Negami expression ${ }^{10}$

$$
\varepsilon^{*}(\omega)=\varepsilon_{\infty}+\frac{\left(\varepsilon_{0}-\varepsilon_{\infty}\right)}{\left[1+(j \omega \tau)^{1-\alpha}\right]^{\beta}}
$$

where $\varepsilon^{*}(\omega)$ is the complex permittivity at an angular frequency $\omega, \varepsilon_{0}$ is the static permittivity, $\varepsilon_{\infty}$ is the permittivity at high frequency, $\tau$ is the relaxation time of the system, $\alpha$ is the shape parameter representing symmetrical distribution of relaxation time and $\beta$ is the shape parameter of an asymmetric relaxation curve. For Debye model, ${ }^{11}(\alpha=0$ and $\beta=1)$ shows the single relaxation and the $\operatorname{CC}^{12}(0 \leq \alpha \leq 1$ and $\beta=1)$ and $\mathrm{CD}^{13}(\alpha=0$ and $0 \leq \beta \leq 1)$ models.

The complex permittivity graph is obtained by plotting dielectric constant $\left(\varepsilon^{\prime}\right)$ versus dielectric loss $\left(\varepsilon^{\prime \prime}\right)$, the shape of this graph indicates type of the model to be adopted.

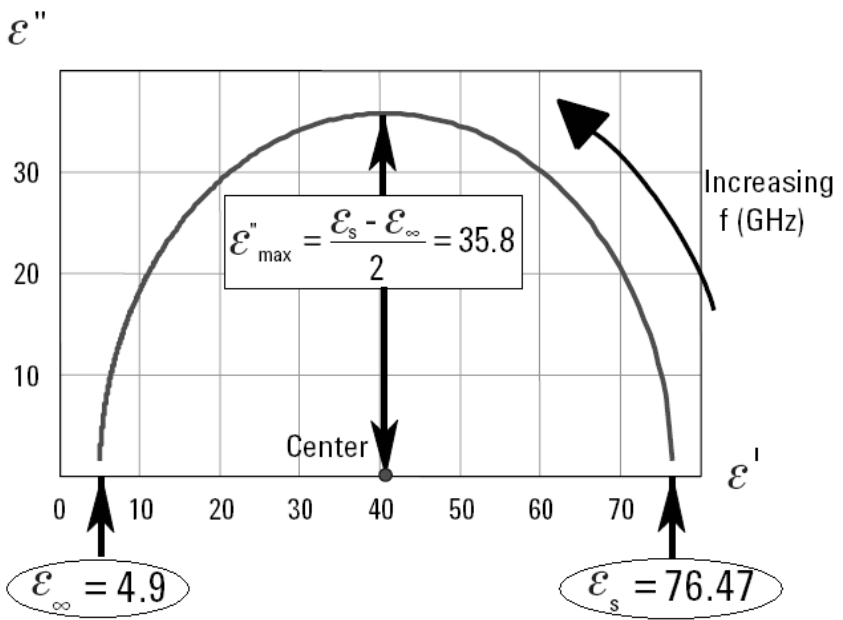

Fig. 2. CC diagram of Debye relaxation.

- Debye model: The shape of the complex plane is in semicircle form, then the dielectric data obey the Debye model as shown in Fig. 2, this model has only one relaxation time $(\tau)$. It gives the complex nature of the dielectric permittivity with loss. The real part complex permittivity indicates orientation effect of dielectric

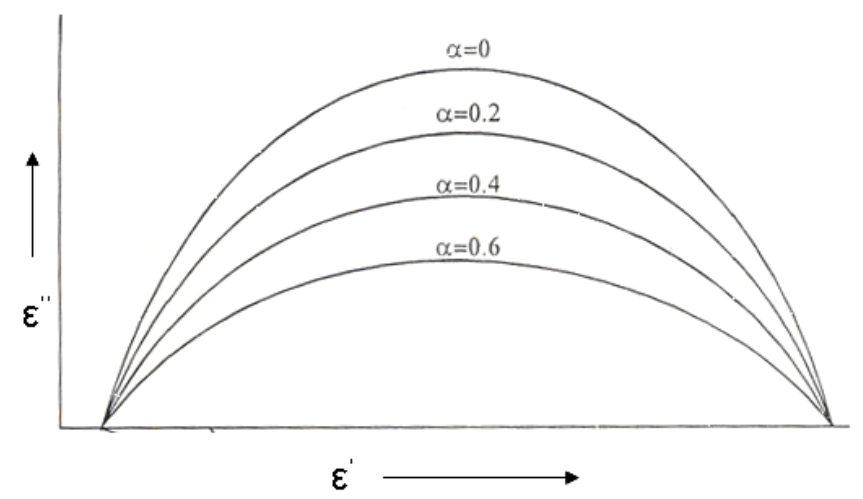

Fig. 3. CC model with different values of symmetric distribution parameter $(\alpha)$.

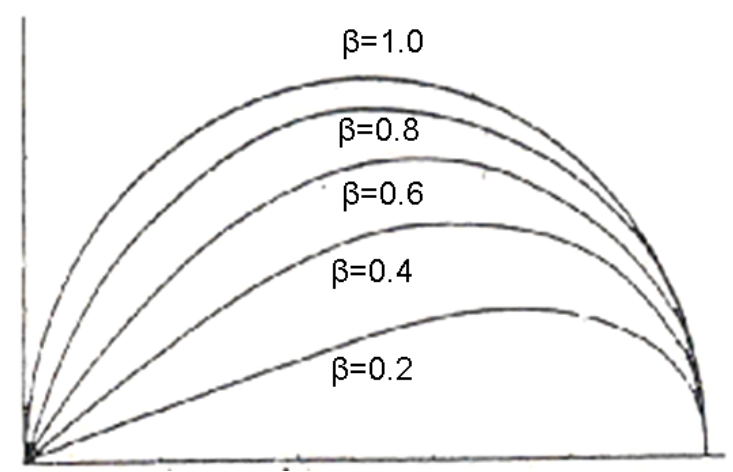

Fig. 4. CC plot for CD model at different $\beta$ values. 


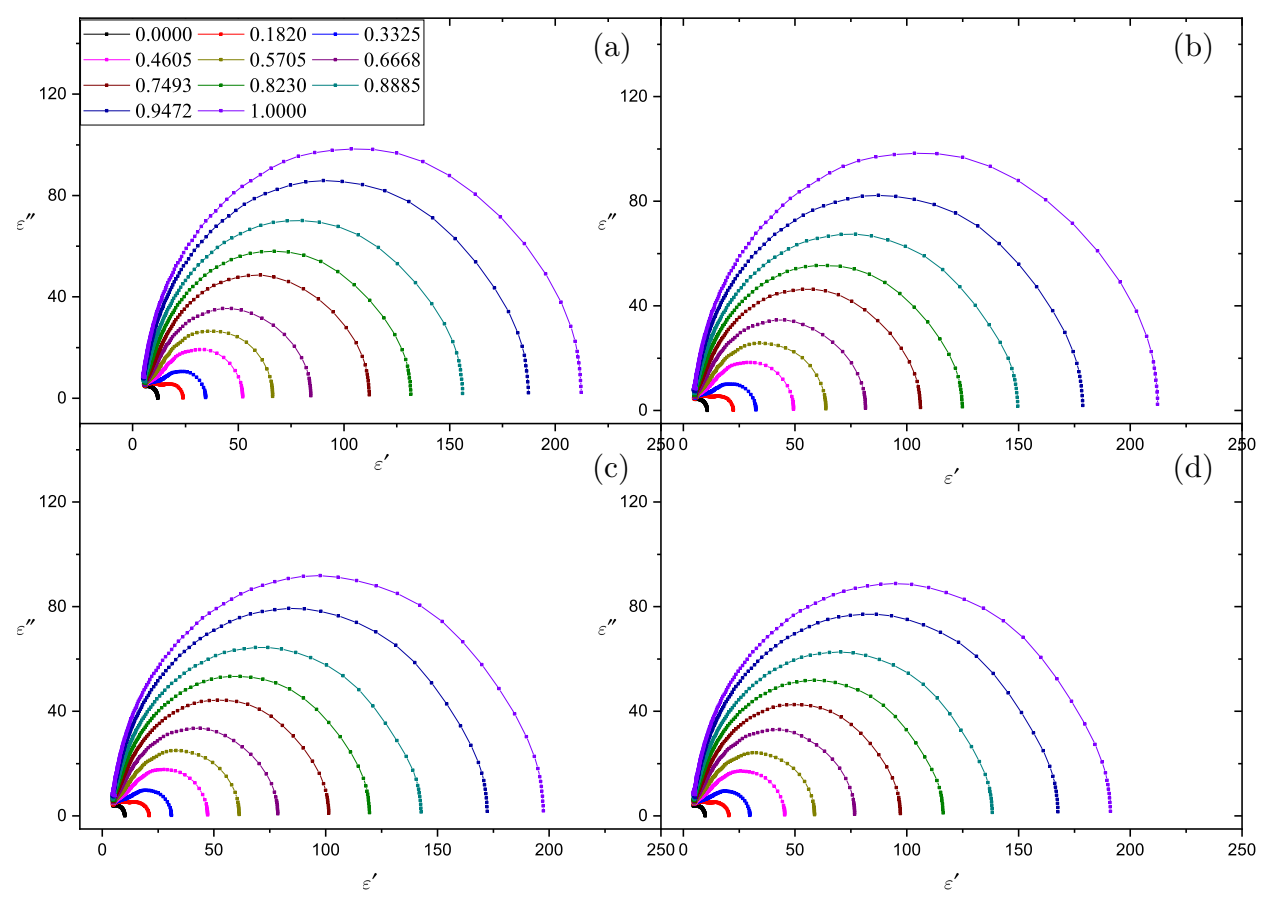

Fig. 5. Complex plane plot of dielectric loss $\left(\varepsilon^{\prime \prime}\right)$ versus dielectric constant $\left(\varepsilon^{\prime}\right)$ for various concentrations of NMF in DE at (a) $T=283 \mathrm{~K}$, (b) $T=288 \mathrm{~K}$, (c) $T=293 \mathrm{~K}$ and (d) $T=298 \mathrm{~K}$.

field with polarization and follows the applied electric field, whereas imaginary part indicates chaotic motion leading to thermal dissipation with opposing the applied field.

- CC model: In this model, binary liquids exhibit boarder dispersion curves and low loss as expected from the Debye model. A material with multiple relaxation frequencies will be indicated by a semicircle (symmetric distribution) or an arc (non-symmetric distribution) with its center lying in the horizontal $\varepsilon^{\prime \prime}=0$ axis as shown in Fig. 3.

- CD model: It describes the symmetric distribution model as represented in Fig. 4. It is corresponding to relaxation time and gives a skewed arc $\varepsilon^{\prime}\left(\varepsilon^{\prime \prime}\right)$. The molecule becomes less rigid with increase in chain length and may relax in many ways.

\section{Results and Discussions}

The complex graph is plotted by taking dielectric dispersion along $x$-axis $\left(\varepsilon^{\prime}\right)$ and dielectric loss along $y$-axis $\left(\varepsilon^{\prime \prime}\right)$ for 11 different concentrations at 283,288, 293 and $298 \mathrm{~K}$ as shown in Fig. 5, this plot is in semicircle shape which indicates that DE-NMF system obeys the Debye's model.

The dielectric properties were studied as a function of logarithmic frequency as shown in Figs. 6-9. The static dielectric constant values are high for low frequencies and the highest value shifted towards right side as the temperature increases and then decreases as expected. The dielectric loss

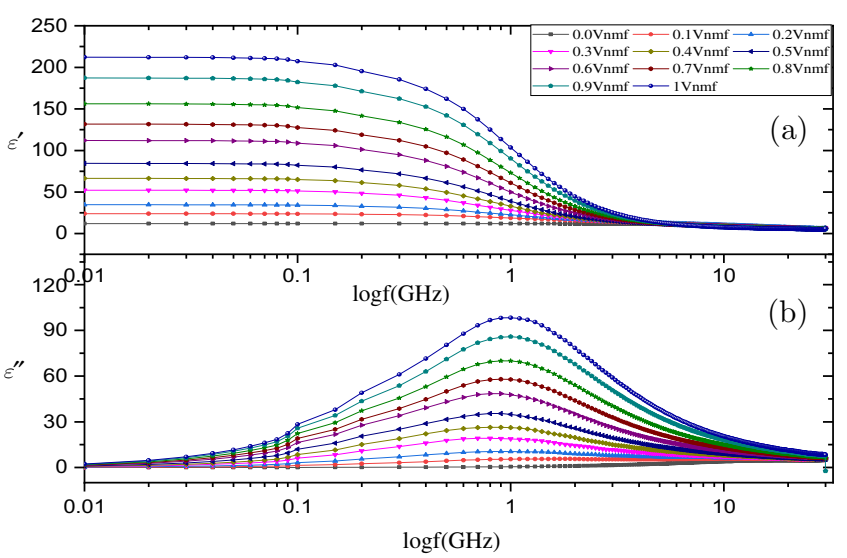

Fig. 6. Complex permittivity spectra $\left(\varepsilon^{\prime}\right.$ and $\left.\varepsilon^{\prime \prime}\right)$ DE-NMF at $283 \mathrm{~K}$.

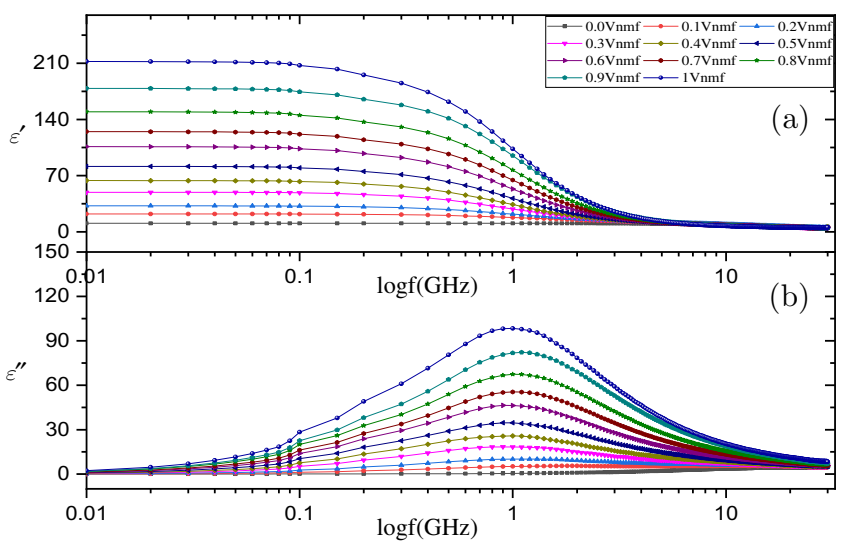

Fig. 7. Complex permittivity spectra $\left(\varepsilon^{\prime}\right.$ and $\left.\varepsilon^{\prime \prime}\right)$ DE-NMF at $288 \mathrm{~K}$. 


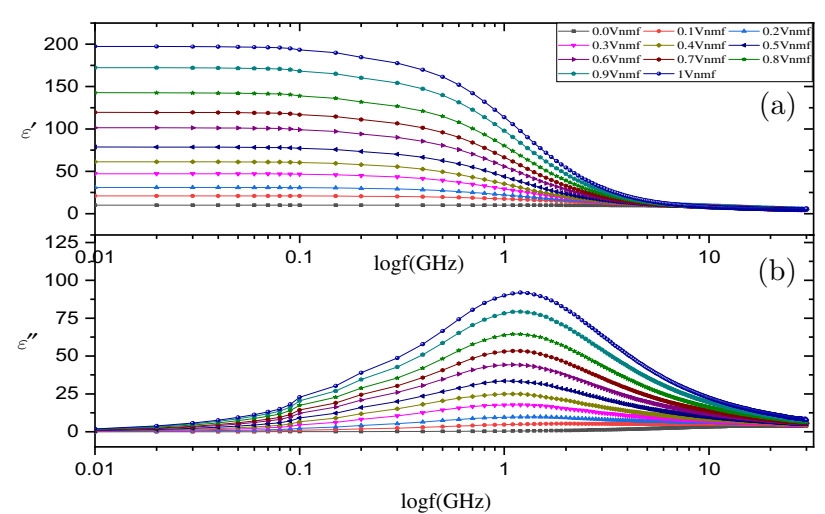

Fig. 8. Complex permittivity spectra $\left(\varepsilon^{\prime}\right.$ and $\left.\varepsilon^{\prime \prime}\right)$ DE-NMF at $293 \mathrm{~K}$.

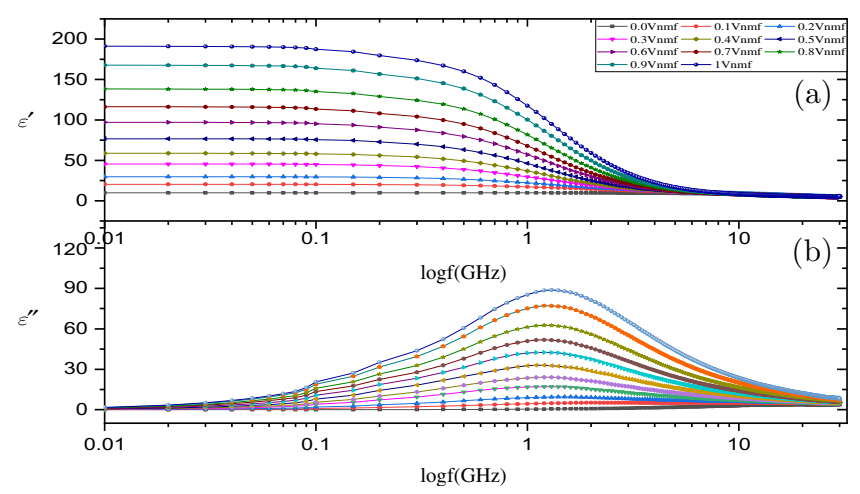

Fig. 9. Complex permittivity spectra $\left(\varepsilon^{\prime}\right.$ and $\left.\varepsilon^{\prime \prime}\right)$ DE-NMF at $283 \mathrm{~K}$. values are less for low applied frequencies and increase with the applied frequency; it shows that the dielectric loss is mainly a function of applied frequency. Only one relaxation peak is observed for pure DE, NMF and DE-NMF binary mixtures since DE-NMF system obeys Debye model. Further, it seems that in the mixture of DE-NMF liquids, the individual principle relaxation processes of the components coalesce, and hence the mixture exhibits a single relaxation time.

The dielectric loss peaks for these molecules and their mixtures are found in the frequency range 1 to $2 \mathrm{GHz}$. The peak of dielectric loss is dependent on the temperature, static loss shifted to the right as the temperature increases.

The static permittivity of mixture DE-NMF of binary polar dielectrics can be described by Bruggeman equation. ${ }^{14}$ It is another important parameter which may be used an indicator of liquid 1 (DE) and 2 (NMF) interactions. The Bruggeman factor $f_{\mathrm{B}}$ is given by

$$
f_{\mathrm{B}}=\left(\frac{\varepsilon_{0 m}-\varepsilon_{02}}{\varepsilon_{01}-\varepsilon_{02}}\right)\left(\frac{\varepsilon_{01}}{\varepsilon_{0 m}}\right)^{1 / 3}=\left(1-\phi_{2}\right) \text {. }
$$

According to the above equation, a linear relationship is expected which gives a straight line when a graph is plotted $f_{\mathrm{B}}$ against $\phi_{2}$ (NMF). However, here the experimental values of $f_{\mathrm{B}}$ were found to deviate from the linear relationship. The Bruggeman dielectric factor $f_{\mathrm{B}}$ versus volume fraction $\phi_{2}$ of NMF at various temperatures is given in Fig. 10.

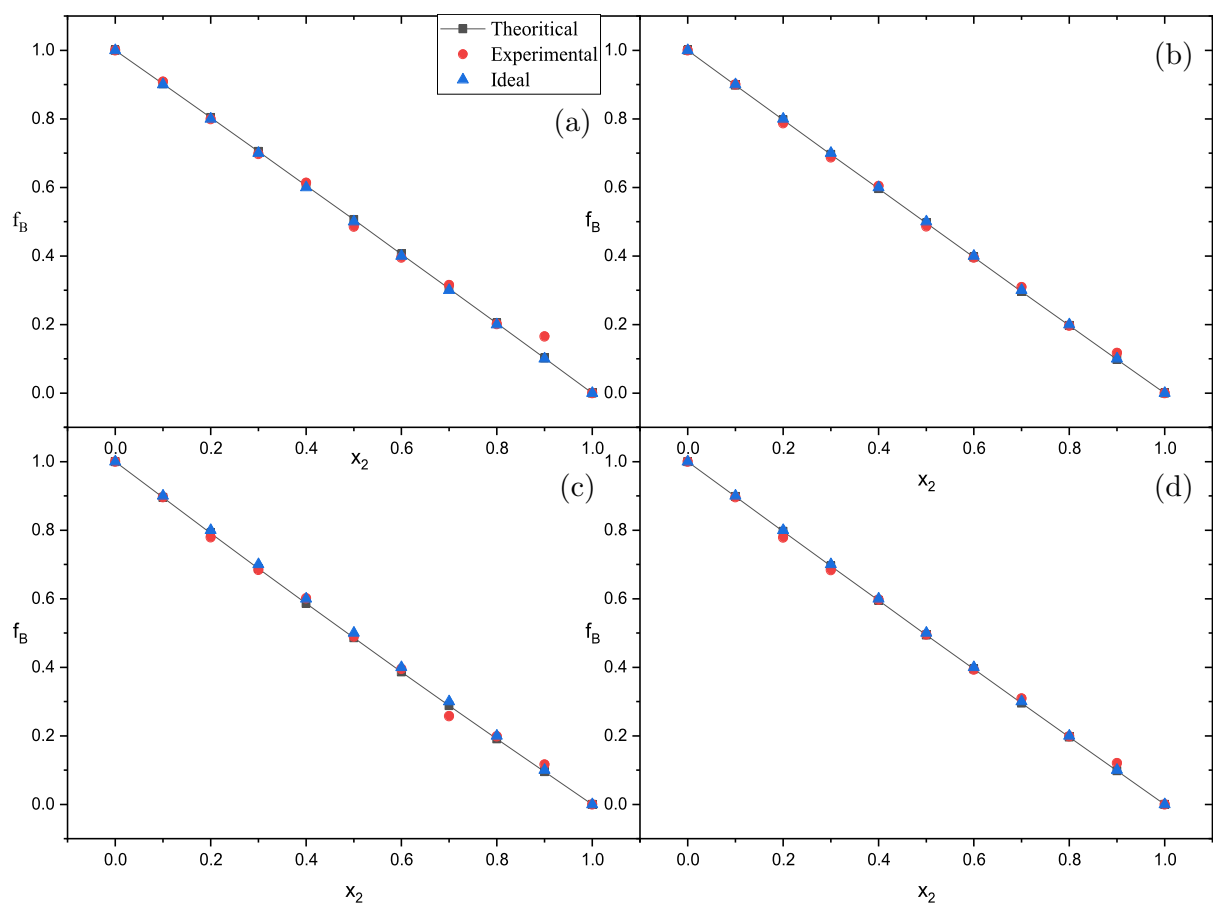

Fig. 10. (Color online) Bruggeman factor $\left(f_{\mathrm{B}}\right)$ versus volume fraction $\left(x_{2}\right)$ of NMF at (a) 283, (b) 288, (c) 293 and (d) $298 \mathrm{~K}$. Solid line represents theoretical Bruggeman model. Blue color dots represent Bruggeman model obtained from Eq. (4). Marker • denotes experimental results. 
Table 1. Values of $a$ for DE-NMF at different temperatures.

\begin{tabular}{lc}
\hline$T(\mathrm{~K})$ & Values of $a$ \\
\hline 283 & 0.978 \\
288 & 1.017 \\
293 & 1.057 \\
298 & 1.023 \\
\hline
\end{tabular}

To fit the experimental data, the above equation has been modified as ${ }^{15}$

$$
f_{\mathrm{B}}=1-\left[a-(a-1) \phi_{2}\right] \phi_{2},
$$

where $a$ is numerical fitting parameter.

The value of $a=1$ corresponds to the ideal Bruggeman mixture formula. The deviation from 1 relates to corresponding liquids 1 and 2 interactions.

The values of $a$ are listed in Table 1 . The values of $a$ indicate the strength of intermolecular interaction for DE-NMF system.
- When $a<1$, it indicates the weak intermolecular interaction.

- When $a>1$, it indicates the strong intermolecular interaction.

The relaxation process in dielectric materials may be described by passing a dipole across a potential barrier that separates the minima of energy. Let $\Delta G$ indicates the difference in free enthalpy per mole of molecules, i.e., the difference of free enthalpy between the excited and ground states. The thermodynamic parameters like molar enthalpy of activation $\Delta H$ and molar entropy of activation $\Delta S$ are determined from the Eyring rate equation ${ }^{16}$ utilizing least square fit method:

$$
\tau=\frac{h}{K T} \exp (\Delta H-T \Delta S) / R T .
$$

In binary mixture of polar liquids mixed together, there is a change in the energy of the system. This change in energy can be interpreted with thermodynamic parameters such as free energy of activation $(\Delta G)$, molar enthalpy of activation $(\Delta H)$ and molar entropy of activation $(\Delta S)$ which are tabulated in

Table 2. Molar enthalpy of activation $(\Delta H)$, molar entropy of activation $(\Delta G)$ and entropy of activation $(\Delta S)$ for DE-NMF system.

\begin{tabular}{lllllll}
\hline$x_{2}$ & $T=283 \mathrm{~K}$ & $T=283 \mathrm{~K}$ & $T=283 \mathrm{~K}$ & $T=283 \mathrm{~K}$ & $\Delta H \mathrm{KJ}$ & $\Delta S \mathrm{KJ}$ \\
\hline \multicolumn{7}{c}{$\Delta G \mathrm{KJ}$} \\
0 & 9.531456 & 9.706369 & 9.881282 & 10.0562 & -0.36863 & -0.03498 \\
0.1 & 10.2796 & 10.43845 & 10.5973 & 10.75615 & 1.28872 & -0.03177 \\
0.2 & 10.33409 & 10.5157 & 10.69732 & 10.87893 & 0.05489 & -0.03632 \\
0.3 & 10.49968 & 10.6225 & 10.74532 & 10.86815 & 3.54789 & -0.02457 \\
0.4 & 10.55252 & 10.69073 & 10.82895 & 10.96716 & 2.7296 & -0.02764 \\
0.5 & 10.53937 & 10.71009 & 10.88081 & 11.05153 & 0.87667 & -0.03414 \\
0.6 & 10.45911 & 10.57792 & 10.69673 & 10.81555 & 3.7343 & -0.02376 \\
0.7 & 10.46163 & 10.54909 & 10.63655 & 10.72402 & 5.51119 & -0.01749 \\
0.8 & 10.29569 & 10.38699 & 10.47828 & 10.56958 & 5.12839 & -0.01826 \\
0.9 & 10.09401 & 10.17798 & 10.26194 & 10.34591 & 5.34163 & -0.01679 \\
1.0 & 10.04445 & 10.11525 & 10.18605 & 10.25685 & 6.03713 & -0.01416 \\
\hline
\end{tabular}

Note: $x_{2}$ represents the mole fraction of NMF in DE.

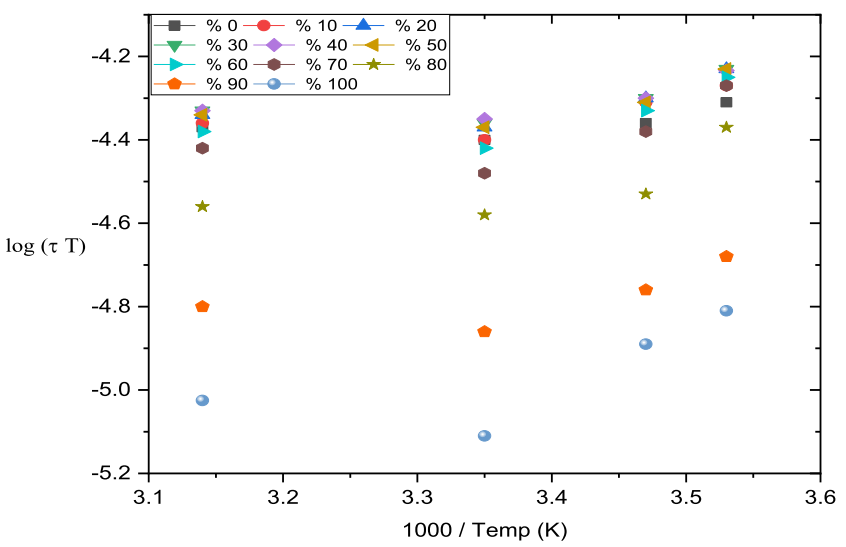

Fig. 11. Arrhenius behavior of DE-NMF system at different temperatures. 
Table 2. The value of free energy of activation $(\Delta G)$ is greater for pure NMF as compared to pure $\mathrm{DE}$ and $\Delta G$ value increases with increase in concentration of NMF at all temperatures. This indicated that the NMF molecules in the mixture dominate over DE molecules. This dominance of NMF over DE is primarily due to its large dipole moment values and presence of $\mathrm{C}=\mathrm{O}$. Plot of $\ln (\tau T)$ versus $1000 / T$ of DE-NMF for various concentrations should be a straight line as shown in Fig. 11. The values of molar enthalpy of activation $(\Delta H)$ are positive for all concentrations except for pure DE of the system. The positive value shows endothermic interaction between DE-NMF mixtures of the system. The values of molar entropy of activation $(\Delta S)$ of the system are negative for all concentrations. It means that the environment of the system is cooperative resulting in the activated state, which is more ordered than the normal state.

\section{Conclusion}

The complex properties of DE-NMF for 11 different concentrations at four different temperatures are observed and also it is noted that DE-NMF system obeys Debye model. Bruggeman parameters are discussed on theoretical, experimental and ideal bases. Thermodynamic properties like molar enthalpy of activation $(\Delta H)$, molar entropy of activation $(\Delta G)$ and entropy of activation $(\Delta S)$ for DE-NMF system are discussed.

\section{Acknowledgments}

The department of Science and Technology, New Delhi is gratefully acknowledged (Project No. SR/S2/LOP-25/ 2007) for its laboratory facility. We are thankful to A. C. Kumbharkhane for providing TDR facility and Dr. S. C. Mehrotra, Ramanujan Chair Professor, Department of Computer Science and IT, Dr. B.A.M. University, Aurangabad for their valuable discussion and suggestions.

\section{References}

${ }^{1}$ E. L. Dreher, T. R. Torkelson and K. K. Beutel, Chloroethanes and chloroethylenes, Ullman's Encylopedia of Industrial Chemistry (Wiley - VCH, Weinheim, 2001).
${ }^{2}$ H. Bipp and H. Kieczka, Formamide, Ullman's Encylopedia of Industrial Chemistry (Wiley - VCH, Weinheim, 2005).

${ }^{3}$ V. P. Pawar, S. Tabassum and A. V. Patil, Structural properties in binary mixtures of polar molecules through microwave dielectric technique, 19th IEEE Int. Conf. Dielectric Liquids (ICDL) (IEEE Explore, Manchester, 2017), pp. 1-4.

${ }^{4}$ V. P. Pawar, A. V. Patil and S. C. Mehrotra, Dielectric relaxation study of acetonitrile with 1,2-dichloroethane using TDR, Int. Conf. Dielectric Liquids (IEEE Explore, Norway, 2011), pp. 1-4. ${ }^{5}$ V. P. Pawar, A. V. Patil, A. R. Patil and S. C. Mehrotra, Dielectric relaxation study of solute-solvent interaction between dimethylene chloride and dimethylformamide using time domain reflectometry, J. Mol. Liq. 155, 16 (2012).

${ }^{6}$ M. B. Swami, V. P. Pawar, P. G. Hudge and A. C. Kumbharkhane, Dielectric relxation study of amines in 2,3-butanediol using picoseconds time domain reflectometry technique, J. Mol. Liq. 190, 178 (2014).

${ }^{7}$ C. E. Shannon, Communication in the presences of noise, Proc. IRE. 37, 10 (1949).

${ }^{8}$ H. A. Samulon, Spectrum analysis of transient response curves, Proc. IRE. 39, 175 (1951).

${ }^{9}$ R. H. Cole, J. G. Berberian, S. Mashimo, G. Chryssikos, A. Burns and E. Tombari, Time domain reflection method for dielectric measurements to $10 \mathrm{GHz}$, J. Appl. Phys. 66, 793 (1989).

${ }^{10} \mathrm{~S}$. Havriliak and S. Negami, A complex plane analysis of $\alpha$-dispersions in some polymer systems, J. Polym. Sci. C 14, 99 (1966).

${ }^{11}$ P. Debye, Polar Molecules (The chemical catalogue company, New York, 1929).

${ }^{12}$ K. S. Cole and R. H. Cole, Dielectric relaxation in glycerine, J. Chem. Phys. 18, 1417 (1950).

${ }^{13}$ D. W. Davidson and R. H. Cole, Dielectric relaxation in glycerine, J. Chem. Phys. 18, 1417 (1950).

${ }^{14}$ D. A. G. Bruggeman, Calculation of various physical constants of heterogeneous substances. I. Dielectric constant and conductivities of the mixed body of isotropic substance, Ann. Phys. 416, 636 (1935).

${ }^{15}$ S. M. Puranik, A. C. Kumbharkhane and S. C. Mehrotra, The static permittivity of binary mixture using an improved Bruggeman model, J. Mol. Liq. 59, 173 (1994).

${ }^{16}$ S. Glasstone, K. J. Laidler and H. Eyring, Theory of Rate Processes (Mc-Graw Hill book Co., New York, 1941). 